\title{
EPIFIL: A DYNAMIC MODEL OF INFECTION AND DISEASE IN LYMPHATIC FILARIASIS
}

\author{
M. S. CHAN, A. SRIVIDYA, R. A. NORMAN, S. P. PANI, K. D. RAMAIAH, P. VANAMAIL, \\ E. MICHAEL, P. K. DAS, AND D. A. P. BUNDY \\ The Wellcome Trust Centre for the Epidemiology of Infectious Disease, Department of Zoology, University of Oxford, Oxford, \\ United Kingdom; Vector Control Research Centre, Medical Complex, Indira Nagar, Pondicherry, India
}

\begin{abstract}
The lack of a quantitative framework that describes the dynamic relationships between infection and morbidity has constrained efforts aimed at the community-level control of lymphatic filariasis. In this paper, we describe the development and validation of EPIFIL, a dynamic model of filariasis infection intensity and chronic disease. Infection dynamics are modeled using the well established immigration-death formulation, incorporating the acquisition of immunity to infective larvae over time. The dynamics of disease (lymphodema and hydrocele) are modeled as a catalytic function of a variety of factors, including worm load and the impact of immunopathological responses. The model was parameterized using age-stratified data collected from a Bancroftian filariasis endemic area in Pondicherry in southern India. The fitted parameters suggest that a relatively simple model including only acquired immunity to infection and irreversible progression to disease can satisfactorily explain the observed infection and disease patterns. Disease progression is assumed to be a consequence of worm induced damage and to occur at a high rate for hydrocele and a low rate for lymphodema. This suggests that immunopathology involvement may not be a necessary component of observed age-disease profiles. These findings support a central role for worm burden in the initiation and progression of chronic filarial disease.
\end{abstract}

Lymphatic filariasis continues to be a significant source of chronic morbidity in the developing world, with more than 120 million people currently thought to be infected with either Wuchereria bancrofti or Brugia malayi, the major lymph-dwelling filariae of humans., ${ }^{1,2}$ Despite the considerable advances in intervention options, ${ }^{3}$ attempts to control the infection have met with variable success, partially because of inadequate understanding of the epidemiology of transmission and disease. ${ }^{4,5}$

This gap in epidemiologic understanding stems from the intricate relationships between infection, immunity and the development of filarial disease. ${ }^{3-7}$ This situation may also reflect the lack of a quantitative framework to assist understanding of the long-term effects and costs of intervention. ${ }^{8}$ Such mathematical frameworks have played an important role in improving understanding of the epidemiology and control of other helminthiases, including intestinal nematodiases, ${ }^{9-11}$ onchocerciasis, ${ }^{12}$ and schistosomiasis. ${ }^{13}$

Simple epidemiologic models, based on the catalytic models devised by Muench, ${ }^{14}$ have been in existence for filariasis since the 1960s. ${ }^{15-17}$ These models have provided useful insights into the dynamics of filariasis in human populations, including improving the understanding of the roles of host immunity and parasite biology in the epidemiology of infection. Less work exists on the modelling of filarial disease, , $, 6,18$ despite the importance of morbidity models in improving understanding of the health impacts of parasite control and thus the ability to rationally evaluate different control options in schistosomiasis. ${ }^{19,20}$

A limitation of previous work has also been that analyses have focussed primarily on the dynamics of infection prevalence (but see Das and others ${ }^{21}$ and Day and others ${ }^{22}$ ). One result is that the relationship between worm burden and the development of filarial morbidity has not yet been quantified. Experience from the modeling of other helminth infections suggests that gaining an understanding of this relationship will be crucial for modeling the development of pathology in filariasis, since morbidity from macroparasitic infections is likely to be related to the parasite load rather than infection prevalence. ${ }^{9}$ Quantification of infection dynamics based on worm intensity will be important for exploring the putative role of host immune responses in the development of disease, particularly of lymphodema. ${ }^{23}$

In this paper, we describe the development of EPIFIL, a deterministic mathematical model for quantifying the relationship between worm population dynamics and morbidity in lymphatic filariasis. Unlike the early catalytic models, EPIFIL is based on a differential equation framework for describing the dynamics of macroparasitic infections. ${ }^{9,24}$ This basic framework is adapted to explicitly link, we believe for the first time, the dynamics of adult filarial worm populations to the age distribution of infection and chronic disease, specifically lymphodema and hydrocele. The model is used to undertake a quantitative assessment of current explanations for the development of pathology in filariasis, including the suggested role of anti-worm immunopathologic responses and the impact of secondary microbial infections. ${ }^{25,26}$

We begin with a brief description of the conceptual framework underlying EPIFIL. The second part of the paper describes the underlying assumptions and formulation of the model, and in the third part of the paper, EPIFIL output is examined and interpreted.

\section{CONCEPTUAL FRAMEWORK}

Infection model. Since infection status in filariasis is commonly assessed indirectly by the presence and density of microfilariae in blood samples, a general mathematical framework for infection dynamics in the host requires the incorporation of the dynamics of two population stages in the parasite's life cycle: the sexually mature adult worm population residing in the lymphatics and the microfilarial population circulating in the peripheral blood. If initially we define the human host population density to be constant on a time scale appropriate to changes in these parasite popu- 
lations, then the simplest formulation is to consider that the dynamics of each worm stage is governed by one gain and one loss term. ${ }^{9}$ For adult parasites, the gain term would portray the recruitment of worms from the vector, while the loss term would denote parasite mortality. Either of these terms may be modulated by immunity, although we assume as a first approximation in this paper from existing evidence regarding the operation of anti-third-stage larvae (L3) immunity both in the field ${ }^{22,27}$ and from animal infections in the laboratory ${ }^{28,29}$ that immunity acts only on the rate of recruitment. For microfilariae, the gain term is simply the female worm reproductive rate while the loss term denotes mortality. We assume again as a first approximation that there is no immunity acting on this stage although this simplifying assumption may well need to be relaxed in future work. Note that this structure to the infection model in EPIFIL affords a great deal of flexibility in addressing current hypotheses regarding infection processes in filariasis. For example, we can define the rate of gain (or the force of infection) to the adult worm populations to either be a constant or a variable function of host age, ${ }^{17}$ and specify the effect and duration of anti-parasite immunity. ${ }^{5,6}$ Note that the model does not explicitly address the biology of larval stages in the vector population, but instead models this by the force of infection parameter.

Throughout the paper, we will refer to the term worm burden, which is the current number of worms in an individual. The accumulated worm burden, on the other hand, is the total number of worms, or specifically, number of worm years and individual has experienced throughout his or her lifetime.

Models for disease. Recent advances from clinical studies have led to a profound change in thinking regarding the pathogenesis of disease in filariasis. There is increasing evidence that all infected individuals may suffer from some worm-induced lymphatic damage, ${ }^{30,31}$ which at least initially is asymptomatic. There is also increasing consensus that progression from this stage to symptomatic disease may occur along different pathways for the two most common manifestations of chronic disease, namely lymphodema and hydrocele. ${ }^{32}$

For lymphodema, the available evidence suggests that pathogenesis could involve three possible mechanisms. The first is that disease develops as a natural sequel to worminduced physical damage to the lymphatics, which eventually becomes sufficient to reduce the flow of lymph fluid. ${ }^{3,31}$ The second is that recurrent host inflammatory reactions to secondary microbial infections picked up through broken skin exacerbate the rate of progression to chronic disease. ${ }^{25,26}$ The third is that the progression to disease occurs as a result of immunopathological reactions to the presence of adult worms. ${ }^{23,33}$ Note that these mechanisms may not be mutually exclusive.

In contrast, the pathogenesis of hydrocele is believed to be more straightforward. The present consensus is that hydrocele represents the progression of lymphatic damage in the scrotal area. ${ }^{34}$ It is thought that early-stage hydroceles may frequently resolve spontaneously.

These considerations imply that mathematical models for filarial disease need to be sufficiently flexible to both incorporate and address the relative merits of these various ex- planations for disease formation. We describe the formal specification and development of these models in the Model development section.

Conceptual framework for model fitting. In this section, the statistical concepts behind the methods used to fit the model are explained. In this paper, we introduce models of varying complexity and use them to infer conclusions about a data set. The models are mechanistic, that is, they describe the processes involved in determining the patterns seen in the data. However, we do not know the mechanisms operating in the actual data. We will use the principle of parsimony, that is, we will choose the simplest, mechanism that can explain the data. This is equivalent to a null hypothesis in statistics; unless the null hypothesis can be disproved, it will be selected. Whether or not a model satisfactorily explains the data is tested by comparison with the saturated model, that is a model that predicts the data observed. In practice, this means testing for a significant difference between the model and the data; if there is a difference, the model is not adequate and a more complex model is required. In this way, using a stepwise process starting with the simplest model, one can find the simplest (most parsimonious) model that can explain the given data. The results do not state that other, more complex mechanisms do not occur, they simply state that more complex mechanisms are not required to explain the data. These concepts are formally described in standard statistical texts..$^{35}$

Two more modeling concepts will be introduced here. First, parameterization is the process of selecting parameter values for a model. This can be done in two ways, both of which are used here. The first method is to use values published elsewhere, the second is to use the parameter value that best fits the data. Since the parameter values may not be known with much accuracy, it is important to look at the consequences if the parameter values were different. Therefore, a process of sensitivity analysis in which parameter values are systematically varied is used to look at the consequences of these.

MODEL DEVELOPMENT

Formal specification. EPIFIL incorporates equations to describe changes by age in worm burden, microfilariae intensity, immunity and prevalences of lymphatic damage (which may be asymptomatic), lymphodema, and hydrocele. It is a cohort model, which means that changes by age of a cohort of individuals in a constant transmission environment are modeled. The structure of the model is shown in Figure 1.

A flow chart, such as that shown in Figure 1, is a diagrammatic representation of the structure of a model. The boxes represent state variables or quantities that describe the situation of the population at a given time. These state variables are mean worm burden (current number of worms per person), mean microfilarial load, mean immunity level, prevalence of lymphatic damage, prevalence of lymphodema, and prevalence of hydrocele in males. An arrow from box $x$ to box $y$ means that $y$ increases at a rate that depends on $x$, with the rate being higher when $x$ is high. If there is a cross on the arrow, $y$ increases faster when $x$ is lower. An arrow leading from a box to nothing means that the quantity in the 


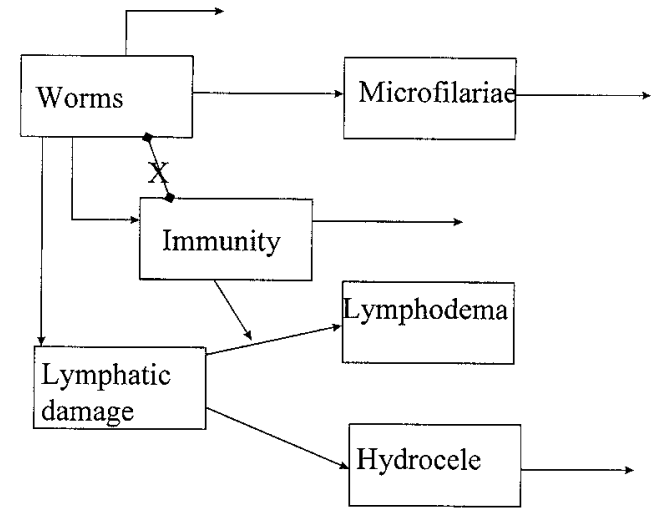

FiguRE 1. Diagrammatic representation of the structure of the model.

box can decrease, e.g., worms can die or hydrocele can resolve.

In the absence of immunity, the rate of gain of worms is a constant (force of infection, $\Lambda$ ). The effect of acquired immunity, I, is to reduce the rate at which the worms enter the host, the degree of reduction being described by a negative exponential function with parameter c. Loss of worms occurs due to the natural death rate of worms, $\mu$, where 1/ $\mu$ is the expected lifespan of a worm. The rate of change of worm burden by age is given by the differential equation

$$
\left.d W / d a=\Lambda \mathrm{e}^{-c I}-\mu W \quad \text { (equation } 1\right) .
$$

It is not currently possible to measure worm burden in the field. Intensity of infection is generally assessed by counting the microfilariae in a sample of blood of known volume, normally, as in this study, via a fingerprick blood smear of $20 \mu \mathrm{l}$. Microfilariae are produced by worms at a constant rate $\alpha$ per year appropriately scaled to reflect the number per blood sample. Microfilariae are lost due to a natural death rate $b$ where $1 / b$ is the expected lifespan. The dynamics of the microfilarial count is given by the equation

$$
d M / d a=\alpha W-b M \quad \text { (equation 2). }
$$

Currently, there are no direct measures of acquired protective immunity available. The model for acquired immunity considered here is intended to reflect the current understanding of the behavior of immune responses in helminth infections. ${ }^{24,36}$ Immunity is assumed to be a consequence of past experience of worms discounted by natural decay, and lasts for $1 / \delta$ years on average. These assumptions can be described by the equation

$$
d I / d a=W-\delta I \quad \text { (equation 3). }
$$

All infected individuals are assumed to develop lymphatic damage that may initially be clinically asymptomatic and is irreversible. All individuals with lymphatic damage are considered to be at risk of developing lyphodema, while males with lymphatic damage are also at risk of developing hydrocele. We model disease states as a set of nonexclusive classes. Thus, D represents the proportion of individuals with lymphatic damage, either asymptomatic or symptomatic. Likewise, L represents the proportion of individuals with lymphodema, regardless of other states and $\mathrm{H}$ represents the proportion of males with hydrocele. The rate of development
TABLE 1

Default parameter values obtained from the published literature*

\begin{tabular}{lccc}
\hline \multicolumn{1}{c}{ Term } & Symbol & Value & Reference \\
\hline Lifespan of worms & $1 / \mu$ & $8 \dagger$ & 49 \\
& & & 50 \\
& & & 51 \\
Lifespan of mf & $1 / \mathrm{b}$ & $1 \dagger$ & 16 \\
& & & 50 \\
Production of mf per worm per & $\alpha$ & 24 & 16 \\
$\quad$ volume of blood & & & \\
\hline$*$ mf = microfilaria. & &
\end{tabular}

of disease states is modeled by catalytic processes. The rate of development of lymphatic damage, assuming no spontaneous resolution, is directly proportional to worm burden:

$$
d D / d a=W(1-D) \quad \text { (equation } 4) .
$$

Lymphodema is assumed to develop via two routes. First, it develops at a constant rate due to direct lymphatic damage and secondary microbial infections at a rate $\pi$. Second, immunologically induced disease progression occurs at a rate $(\phi)$ that is proportional to the level of immunity. Again there is assumed to be no spontaneous resolution:

$$
d L / d a=(\pi \mathrm{D}+\phi D I)(1-L) \quad(\text { equation } 5) .
$$

Hydrocele in males is assumed to develop at a constant rate $h$ and resolve spontaneously at a constant rate $r$ implying the following equation:

$$
d H / d a=h D(1-H)-r H \quad \text { (equation 6). }
$$

Of the parameters above, $\Lambda$, c, and $\delta$ are estimated by fitting to the infection data. The parameters $\pi, \phi, r$, and $h$ are estimated using disease data, and $\mu, \alpha$, and $b$ are taken from the literature and are given in Table 1.

Sensitivity analysis. To explore the patterns of behavior of EPIFIL, simulations were run in which the parameters were varied. The behavior of the simple immigration death model used to describe the intensity of infection by age allowing for acquired immunity is well described in the literature..$^{9,24,36}$ However, the description of the dynamics of disease prevalence in EPIFIL is a new formulation, and therefore sensitivity analysis was carried out on the four disease parameters. A simulation set was devised in which one parameter was varied at a time with the other three kept constant. The results are represented graphically to depict the influence of changing each parameter. The four parameters that were varied are the rate of development of lymphodema $(\pi)$, the immunologic component of the development of lymphodema $(\phi)$, the rate of development of hydrocele (h), and the rate of resolution of hydrocele (r). Default parameter values were chosen to give disease curves which reflect those seen in the field. The specific parameter values are shown in Figure 2.

Parameterization. The parameters of the model were estimated using epidemiologic data collected in Pondicherry $\left(12^{\circ} \mathrm{N}, 80^{\circ} \mathrm{E}\right)$ in southern India. Infection data are from a precontrol survey undertaken in $1981^{37}$ with a $10 \%$ age- and area-stratified sample $(n=24,677)$ of the entire population of Pondicherry. Microfilarial counts in a night blood smear of $20 \mu \mathrm{l}$ were measured for each individual. 

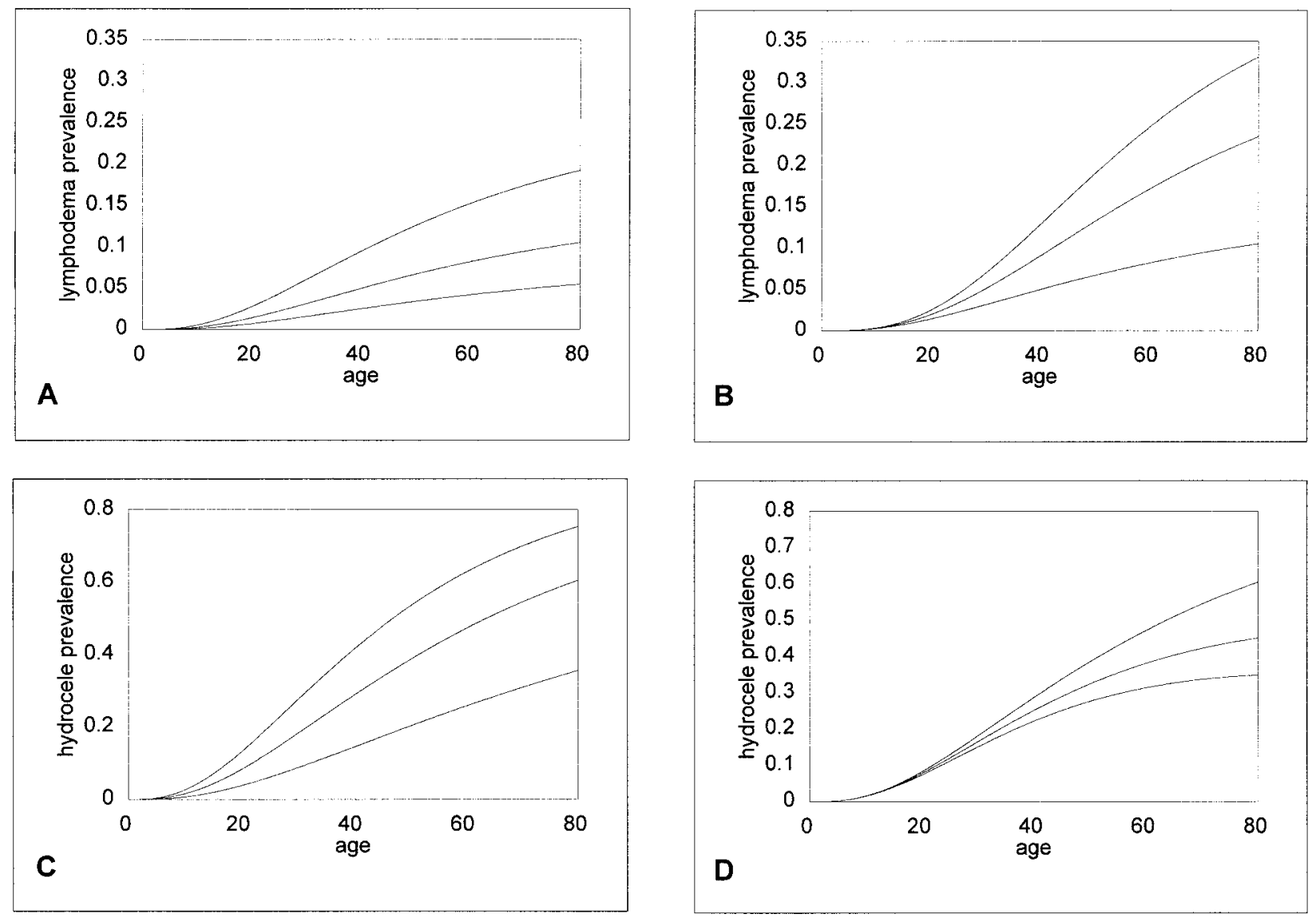

FIGURE 2. Sensitivity analysis of the disease parameters. The default parameter set used is $\Lambda=0.0066, \mathrm{c}=0.2, \delta=0, \pi=0.0047, \phi=$ $0, \mathrm{~h}=0.032$, and $\mathrm{r}=0$. One parameter is varied in each graph, with the curves representing from bottom to top in $\mathbf{A}$ the prevalence of lymphodema with $\pi=0.002, \pi=0.0047$, and $\pi=0.008$; in $\mathbf{B}$ the prevalence of lymphodema with $\phi=0, \phi=0.004$, and $\phi=0.008$; in $\mathbf{C}$ the prevalence of hydrocele with $\mathrm{h}=0.01, \mathrm{~h}=0.032$, and $\mathrm{h}=0.04$; and in $\mathbf{D}$ the prevalence of hydrocele with $\mathrm{r}=0.02, \mathrm{r}=0.01$, and $\mathrm{r}=$ 0 . Age is in years.

Disease data (age prevalences of hydrocele and lymphodema) are from a clinical survey carried out in 1986 on a sample of 6,493 individuals in Pondicherry. ${ }^{38}$ It is assumed that the chronic disease patterns changed little between the infection and disease surveys, an assumption supported by the observation that these disease states develop over many decades of infection. The data for all grades of lymphodema and for males and females combined were considered. The data for hydrocele are for males only and all grades combined.

Infection and disease data were fitted separately. The infection model (although describing changes in mean worm burden) was fitted using microfilariae prevalence since this was considered a more reliable indicator because it has a lower variance and therefore can be estimated with greater accuracy. ${ }^{39}$ The first step was therefore to quantify the relationship between prevalence and mean intensity. This was done by plotting these measures for the 19 zones of Pondicherry, and fitting a negative binomial model to quantify the observed prevalence-mean intensity relationship using the maximum likelihood method. ${ }^{40}$ The estimated relationship was then used to predict age prevalence curves from the corresponding mean intensity changes described by the infection model (equation 2). These curves were then fitted to the observed data by maximum likelihood, assuming binomial errors. ${ }^{40}$ The curve was initially fitted using the simple immigration-death model with a constant force of infection. This involves fitting three parameters, the force of infection $(\Lambda)$, the strength of immunity (c) and the duration of immunity $(\delta)$. The model was also fitted with a variable force of infection, which was assumed to increase linearly to the age of 10 and then remain constant. This pattern of exposure is consistent with rates of gain of infection from contemporary data from Pondicherry. ${ }^{17}$ The relationship between mean microfilariae count and age was plotted to assess the consistency of the model.

In fitting the infection profile, one question of interest was whether the data provided any evidence for the operation of acquired immunity. In the absence of acquired immunity, and if the force of infection does not decrease with age, only monotonically increasing or asymptotic infection (prevalence or intensity) curves can be expected. ${ }^{9}$ A decrease in intensity or prevalence of infection in older ages implies that either acquired immunity is having an effect or that the force of infection decreases at older ages. These considerations were tested by fitting the model sequentially with the assumptions of no immunity, life-long immunity, and immunity that can decay with time. Each of these successive mod- 


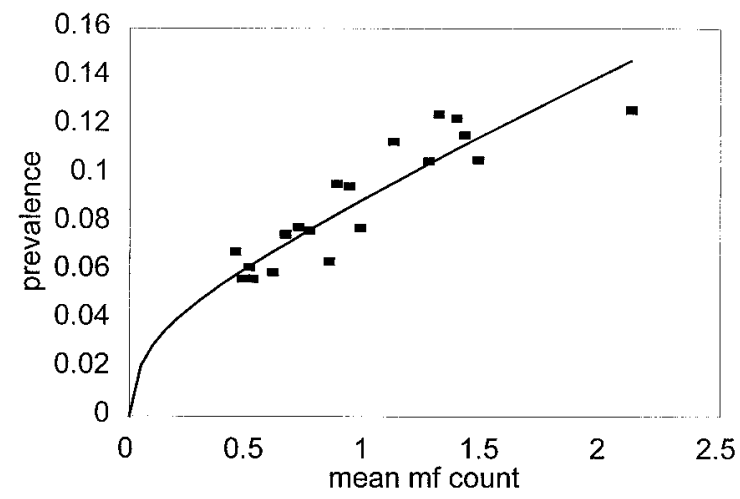

FIGURE 3. Plot of prevalence of microfilaria ( $\mathrm{mf}$ ) in blood versus mean mf count. The squares show the data for each of the 19 zones in Pondicherry, and the line shows the fitted negative binomial model (see Results).

els involve one more parameter resulting in the loss of one degree of freedom. The statistical significance of the increase in likelihood can then be tested using the chi-square statistic.

Using the results of the fitted infection model, the disease part of EPIFIL was then fitted to data on the prevalence of lymphodema and hydrocele. Again, this was done sequentially to determine the most parsimonious model, starting with the simplest model in which there is no immunologically induced pathology or spontaneous resolution and then adding the other parameters one by one. This was done separately for hydrocele and lymphodema. For hydrocele, the simplest model involved a constant rate of development with no resolution, while the second model included the resolution of the disease state. Similarly, for lymphodema, the simplest model involved a constant rate of development, whereas the second model also included the immunopathologic development of the disease.

\section{RESULTS}

Sensitivity analysis. The results of the sensitivity analysis are shown in Figure 2. It can be seen that the prevalence of lymphodema increases very slowly with age, being almost absent in the first 20 years of life and thereafter increasing to a prevalence of less than $20 \%$ by age 80 years in most of the simulations. This is in accordance with observation in the field. In contrast, the prevalence of hydrocele in males tends to appear earlier in life and also reach much higher prevalences by age 80 years (30-80\%). This suggests that a significant proportion of adult males in an endemic population may have hydrocele. This is supported by available field data. ${ }^{37,41}$

As the rate of development of lymphodema is increased (Figure 2A), the prevalence of lymphodema at all ages also increases by approximately the same relative amount. If some immunologically induced lymphodema is added to the physically induced lymphodema (Figure 2B), the prevalence of lymphodema increases but disproportionately in the older age groups, such that the rate of development of lymphodema increases with age (this is due to the DI term in equation 5, which means essentially that lymphodema increases as the square of accumulated worm burden experience). Thus, a predominance of lymphodema in older age groups, exceeding that which could be explained by a constant rate of development, may imply that immunopathologic processes are involved.

Increasing the rate of development of hydrocele increases its prevalence (Figure 2C), although the prevalence in older age groups may not increase as rapidly if there is some degree of spontaneous resolution (Figure 2D). If there is resolution of hydrocele, the age prevalence curves tend to reach an equilibrium between the rates of development and resolution, leading to a constant proportion with hydrocele in older age classes irrespective of age.

Parameterization. Infection parameters. Figure 3 shows the relationship of prevalence with mean microfilariae count. The fit of the negative binomial model ${ }^{40}$ indicates that the parameter of dispersion, $k$, is a linear function of the mean microfilariae count, with maximum likelihood estimates of the intercept $\left(\mathrm{k}_{0}\right)$ and slope $\left(\mathrm{k}_{\text {lin }}\right)$ being 0.013 and 0.0126 , respectively. The low values suggest that the distribution of counts is highly aggregated and the strong linear term suggests that this aggregation is particularly strong at a low mean intensity of infection, which is in agreement with previous analyses for a variety of helminth species. ${ }^{40}$

The maximum likelihood analysis of the infection model is shown in Table 2. The results show that the model with a variable force of infection gives a much better fit to the data than the model with a constant force of infection. Adding immunity to the variable force of infection model gives a significantly better fit $(P<0.001)$. If the immunity is allowed to decay, the fit improves but is not significant. Therefore, the most parsimonious model is the one in which there

TABLE 2

Analysis of models of infection

\begin{tabular}{|c|c|c|c|c|c|}
\hline Model & $\Lambda$ & $\mathrm{c}$ & $\delta$ & $\begin{array}{c}\text { Log } \\
\text { likelihood }\end{array}$ & $P^{*}$ \\
\hline \multicolumn{6}{|l|}{ 1) Constant force of infection } \\
\hline $\begin{array}{l}\text { 1a) No immunity } \\
\text { 1b) With immunity }\end{array}$ & $\begin{array}{l}0.0056 \\
0.0063\end{array}$ & $\begin{array}{l}0 \\
0.17615\end{array}$ & $\begin{array}{l}0 \\
0\end{array}$ & $\begin{array}{l}-3080.3 \\
-3078.6\end{array}$ & NS \\
\hline \multicolumn{6}{|l|}{ 2) Variable force of infection } \\
\hline $\begin{array}{l}\text { 2a) No immunity } \\
\text { 2b) With life-long immunity } \\
\text { 2c) Immunity with decay } \\
\text { Saturated model }\end{array}$ & $\begin{array}{l}0.0065 \\
0.0092 \\
0.0097\end{array}$ & $\begin{array}{l}0 \\
0.51948 \\
0.80739\end{array}$ & $\begin{array}{l}0 \\
0 \\
0.0235\end{array}$ & $\begin{array}{l}-3078.0 \\
-3065.3 \\
-3064.8 \\
-3061.2\end{array}$ & $\begin{array}{l}<0.001 \\
\text { NS }\end{array}$ \\
\hline
\end{tabular}

$* P$ is given with respect to the appropriate simplest model i.e., $1 \mathrm{a}$ or $2 \mathrm{a}$. Models $2 \mathrm{~b}$ and $2 \mathrm{c}$ are not significantly different from the saturated model. NS $=$ not significant. 


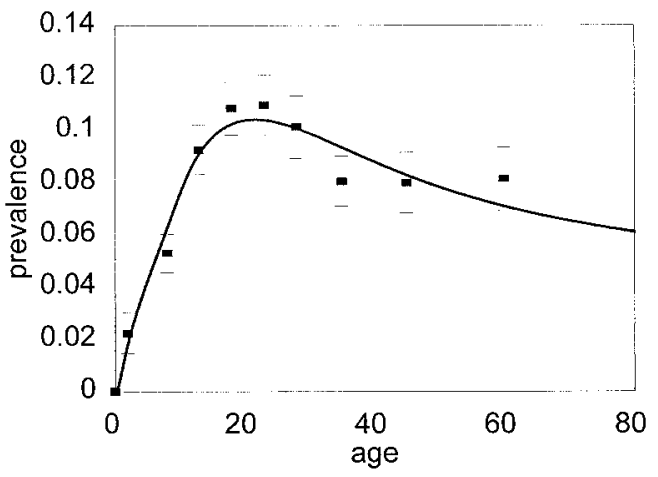

FigURE 4. Fitted model for prevalence of infection (model $2 \mathrm{~b}$ in Table 2). The squares show the data points with $95 \%$ confidence intervals and the line shows the fitted model. Age is in years.

is life-long immunity. This model is also not significantly different from the saturated model.

The most parsimonious model is plotted against prevalence data in Figure 4, and shows good correspondence with the curve falling within the confidence intervals of the data. This graph shows the prevalence increasing to a peak at age 20 and decreasing slightly thereafter. The model also corresponds well with data on mean microfilariae count (Figure 5), which although more variable, shows a similar peak at age 20 years.

Disease parameters. The maximum likelihood analysis of the disease model is shown in Table 3. For hydrocele, the simplest model with no resolution is not significantly different from the saturated model. For lymphodema also, the simplest model with no immunologically induced development of lymphodema is not significantly different from the saturated model. This analysis shows that the simplest model is already an adequate description of the data and that addition of the other parameters does not significantly improve the fit. This implies that the data can be explained by a model in which both hydrocele and lymphodema are irreversible conditions that develop as a consequence of lymphatic damage caused by worms, with the risk of disease being higher for hydrocele than lymphodema. Immunologic involvement is not necessary to explain these data although the data do not preclude the existence of other mechanisms. Note that

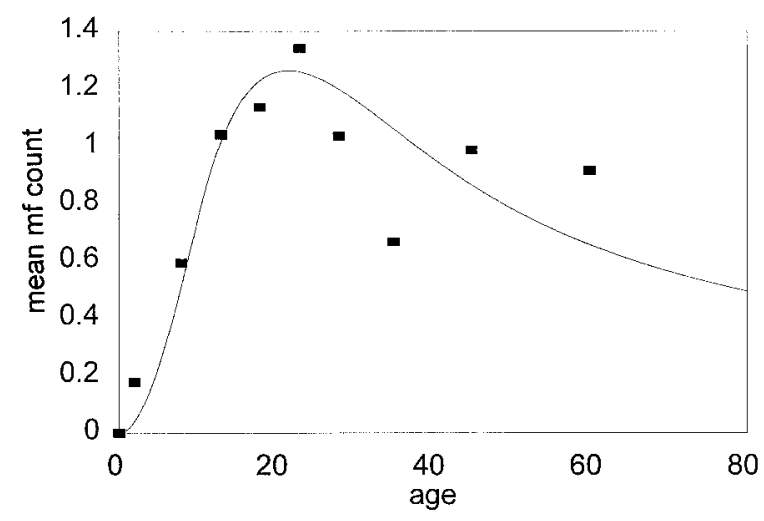

FigURE 5. Fitted model for mean microfilaria (mf) count where the squares show the data points and the line shows the fitted model. Age is in years.
TABLE 3

Analysis of models of disease*

\begin{tabular}{lcccc}
\hline \multicolumn{1}{c}{ Model } & $\begin{array}{c}\text { Parameter } \\
1\end{array}$ & $\begin{array}{c}\text { Parameter } \\
2\end{array}$ & \multicolumn{1}{c}{$\begin{array}{c}\text { Log } \\
\text { likelihood }\end{array}$} & $P_{\dagger}^{\dagger}$ \\
\hline 1) Hydrocele, parameter $1=\mathrm{h}$, parameter 2 & $\mathrm{r}$ & \\
1a) $\mathrm{r}=0$ & 0.022 & 0 & -266.6 & \\
1b) $\mathrm{r} \geq 0$ & 0.032 & 0.024 & -264.72 & $\mathrm{NS}$ \\
Saturated model & & & -262.2 & \\
2) Lymphodema, parameter $1=\pi$, parameter & $=\phi$ & \\
2a) $\phi=0$ & 0.0033 & 0 & -249.54 & \\
2b) $\phi \geq 0$ & 0.0033 & 0 & -249.54 & NS \\
Saturated model & & & -245.14 & \\
\hline
\end{tabular}

$*$ None of the models are significantly different from the respective saturated model. The $P$ value for the difference between models $1 \mathrm{a}$ and $1 \mathrm{~b}$ is between 0.05 and 0.1 .

$\dagger \mathrm{NS}=$ not significant.

the difference between the model for hydrocele with and without resolution is significant at the 0.1 level, which suggests the possibility that hydrocele does resolve spontaneously.

The most parsimonious model is shown in Figure 6 and indicates a good fit between model and data. This model has only two parameters related to disease: the rate of development of hydrocele ( 0.022 per year) and the rate of development of lymphodema ( 0.0033 per year), where the rates are related to accumulated experience of worms (and associated lymphatic damage). These rates can be interpreted as a risk of developing disease, per worm-year of infection experienced $(2.2 \%$ per worm-year for hydrocele and $0.33 \%$ per worm-year for lymphodema). Thus, for example, one worm experienced for one year leads to an increased risk of developing hydrocele of $2.2 \%$, and a worm over its lifetime (eight years) will increase the risk by $17.6 \%$. The corresponding figures for lymphodema are $0.33 \%$ and $2.64 \%$.

\section{DISCUSSION}

Previous models of filarial disease $\mathrm{e}^{5,6,18}$ have been based on the concept that individuals progress from uninfected to the asymptomatic microfilaremic condition and then from the

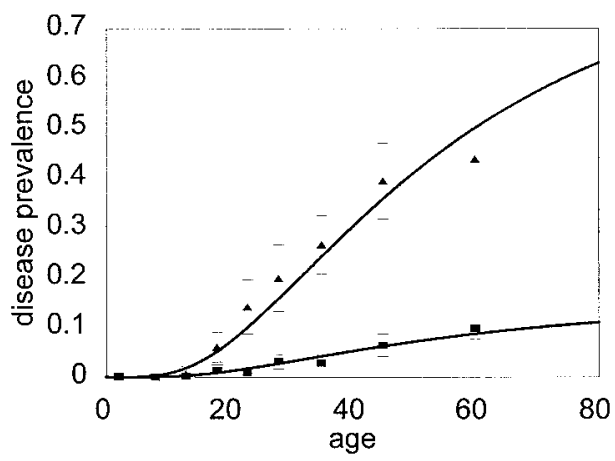

FiguRE 6. Fitted model for disease (models 1a and 2a in Table $3)$. The top line shows the predicted prevalence of hydrocele in males with the triangles representing the data points and the bars the corresponding upper and lower $95 \%$ confidence intervals. The lower line is the predicted prevalence of lymphodema. The squares denote the observed data while the bars show the respective $95 \%$ confidence intervals. When the observed number with disease was less than 10, exact binomial confidence intervals were calculated, otherwise the normal approximation was used. Age is in years. 
symptomatic amicrofilaremic condition (and at risk of disease) to showing disease symptoms. Thus, implicit in these prevalence-based models is the assumption that microfilaremia either protects against disease or that the loss of microfilariae somehow induces disease, models that originate from immunologic work. ${ }^{23,28,33,42}$ EPIFIL, in contrast, draws on the simpler assumption that worms cause damage to the lymphatic system and thus progression to disease. As well as its intuitive biological appeal, this simple model is supported by experimental observations using ultrasound that worms in the body do cause lymphatic damage even before clinical symptoms are observed..$^{3,30}$ It is also pertinent here to note that this formulation of disease development is in line with the analyses of a range of other helminthiases, which indicate that morbidity due to helminths is a function of worm load. ${ }^{43,44}$

Statistical analysis of the data using maximum likelihood methods has led to several results of biological interest. The age-infection data suggest the presence of acquired protective immunity. That is, immune responses induced by the presence of worms and resulting in a reduced rate of establishment of new infections (as opposed to immunity related to disease). The analysis also suggests that this protective response leads to only partial protection, a feature that is common to most helminth infections. ${ }^{45}$ However, immunologic theories of filarial infection have often advocated more complex mechanisms, such as maternal tolerance, ${ }^{46}$ the protective effects of microfilariae, as well as a variety of $\mathrm{T}$ cellmediated responses. ${ }^{33}$ While not precluding these mechanisms, the current results indicate that the observed epidemiologic patterns, at least for southern India, may be equally well explained by a much simpler mechanism.

The analyses also suggest that the development of hydrocele is simply related to the past experience of worms (and the damage they cause). This is in agreement with current thinking in this area. We further estimate that a single worm (over its lifetime) will increase the host risk of progressing to hydrocele by an absolute amount of $17.6 \%$. Given that typical worm burdens are likely to consist of several tens of worms (Dreyer G, unpublished data), this suggests that most infected males will eventually develop hydrocele, which is reasonable given the high prevalences observed in many endemic areas. ${ }^{38,41}$ The data show only very weak evidence $(P$ $<0.1$ ) of a significant resolution of hydrocele. This is consistent with clinical studies, which indicate that the resolution of hydroceles depends on their size, with spontaneous resolution being observed only with small hydroceles. ${ }^{41,47}$

For progression to lymphodema, the analysis suggests that the most important factor, as with hydrocele, is simply the presence of worms. The data do not provide strong evidence for the involvement of immunopathologic responses. The estimated level of risk is small; $2.64 \%$ per worm over its lifetime. The apparent lack of a role for immune responses is somewhat surprising given the observed correlations of lymphodema with various immunologic measures, ${ }^{33}$ although the quantitative contribution of these factors to the determination of disease is unknown. Note that EPIFIL is currently unable to distinguish between lymphodema induced by lymphatic damage alone and that induced in addition by secondary bacterial infections. ${ }^{25,26}$ The results also suggest that lymphodema does not resolve on a significant scale.
In understanding the results of these analyses and why they sometimes appear to differ from experimental observations, it is important to understand the context in which effects are said to be significant. In this study, we are interested in explaining patterns observed on a population level and the major factors that determine these patterns. We aim to explain the general age-related patterns of infection and disease and, eventually, the impact of treatment on these patterns. The analyses suggest that these patterns can be explained by a relatively simple model including only acquired immunity to infection and irreversible progression to disease, which occurs as a consequence of worm-induced damage at a high rate for hydrocele and a low rate for lymphodema. We are not aiming to explain the diverse factors that will undoubtedly cause variations in the way disease progresses in individual patients. When this is taken into account, inevitably several more factors, including immunologic status, are likely to become important.

This paper has fulfilled two objectives. First, using previous ideas from both filariasis ${ }^{5,6,18}$ and schistosomiasis, ${ }^{13,19,20}$ we have developed an epidemiologic model called EPIFIL that adequately (in a statistical sense) describes the age-dependent patterns of filarial infection intensity and disease observed in an endemic area. Second, we have parameterized this model, which will aid the ongoing work to develop a model to predict the impact of intervention (vector control and community chemotherapy) on mean levels of infection and disease in the community. Future work will also attempt to validate and assess the robustness of the present findings by fitting the models to data from a range of endemic areas differing in both transmission intensity and vector species. In particular, this will provide further clarification concerning the importance of immunopathologic involvement in the progression of clinical disease since recent work has shown that immunity is likely to be greater in areas with higher transmission. ${ }^{48}$ This work will also aim to assess the impact of blood sampling volumes on the estimation of infection since it is possible that such sampling artifacts may modify the relationships observed between infection and disease in different studies.

Epifil. Epifil is a collaborative project between the Wellcome Trust Centre for the Epidemiology of Infectious Disease (WTCEID), the Vector Control Research Centre, and the University of Stirling. The aim of this project is to develop mathematical models to aid in the design of filariasis control programs. Initially, these models will be used to assess different control options in Pondicherry. Epifil models will also be available as software to interested individuals. The model described in this paper is available in spreadsheet form (Microsoft Excel for Windows 95) with documentation at the WTCEID web site at http://www.ceid.ox.ac.uk/download/ where it is available for downloading. Enquiries about this program and other Epifil models can be addressed to $\mathrm{M}$. S. Chan.

Acknowledgments: We express our gratitude to the late Dr. Vijay Dhanda (Director, Vector Control Research Centre) for his interest and active encouragement of this study. We are also grateful to the World Health Organization for organizing a consultative meeting of filariasis experts in 1996 on the topic of modeling filarial infection, disease and control, which proved invaluable in developing the structure of the present model. 
Financial support: R. A. Norman and D. A. P. Bundy acknowledge the support of the Wellcome Trust. M. S. Chan is a recipient of a Wellcome Trust Training Fellowship. E. Michael is in receipt of a Medical Research Council Career Development Fellowship. This study was supported by the Wellcome Trust.

Authors' addresses: M. S. Chan, E. Michael, and D. A. P. Bundy, The Wellcome Trust Centre for the Epidemiology of Infectious Disease, Department of Zoology, University of Oxford, South Parks Road, Oxford OX1 3PS, United Kingdom. A. Srividya, S. P. Pani, K. D. Ramaiah, P. Vanamail, and P. K. Das, Vector Control Research Centre, Medical Complex, Indira Nagar, Pondicherry 605 006, India. R. A. Norman, Department of Computing Science and Mathematics, University of Stirling, Stirling FK9 4LA, United Kingdom.

\section{REFERENCES}

1. Michael E, Bundy DAP, Grenfell BT, 1996. Re-assessing the global prevalence and distribution of lymphatic filariasis. Parasitology 112: 409-428.

2. Michael E, Bundy DAP, 1997. Global mapping of lymphatic filariasis. Parasitol Today 13: 472-476.

3. Ottesen EA, 1994. The human filariases: new understandings, new therapeutic strategies. Curr Opin Infect Dis 7: 550-558.

4. Southgate BA, 1984. Recent advances in the epidemiology and control of filarial infections including entomological aspects of transmission. Trans R Soc Trop Med Hyg 78: 19-28.

5. Bundy DAP, Grenfell BT, Rajagopalan PK, 1991. Immunoepidemiology of lymphatic filariasis: the relationship between infection and disease. Immunoparasitol Today (suppl): A71A75.

6. Grenfell BT, Michael E, 1992. Infection and disease in lymphatic filariasis: an epidemiological approach. Parasitology 104: S81-S90.

7. Michael E, Grenfell BT, Bundy DAP, 1994. The association between microfilaraemia and disease in lymphatic filariasis. Proc R Soc Lond B Biol Sci 256: 33-40.

8. Michael E, Meyrowitsch DW, Simonsen PE, 1996. Cost and cost effectiveness of mass diethylcarbamazine chemotherapy for the control of bancroftian filariasis: comparison of four strategies in Tanzania. Trop Med Int Health 1: 414-426.

9. Anderson RM, May, RM, 1992. Infectious Diseases of Humans. Oxford: Oxford University Press.

10. Medley GL, Guyatt HL, Bundy DAP, 1993. A quantitative framework for evaluating the effect of community treatment on the morbidity due to ascariasis. Parasitology 106: 211221.

11. Chan MS, Guyatt HL, Bundy DAP, Medley GF, 1994. The development and validation of an age structured model for the evaluation of disease control strategies for intestinal helminths. Parasitology 109: 389-396.

12. Plaisier AP, van Oortmarssen GJ, Habbema JDF, Remme J, Alley ES, 1990. ONCHOSIM: a model and computer simulation program for the transmission and control of onchocerciasis. Comp Meth Prog Biomed 31: 43-56.

13. Chan MS, Guyatt HL, Bundy DAP, Booth M, Fulford AJC, Medley GF, 1995. The development of an age structured model for schistosomiasis transmission dynamics and control and its validation for Schistosoma mansoni. Epidemiol Infect 115: 325-344.

14. Muench H, 1959. Catalytic Models in Epidemiology. Cambridge, MA: Harvard University Press.

15. Hayashi S, 1962. A mathematical analysis on the epidemiology of bancroftian and malayan filariasis in Japan. Jpn J Exp Med 32: $13-43$.

16. Hairston NG, Jachowski LA, 1968. Analysis of the Wuchereria bancrofti population in the people of American Samoa. Bull World Health Organ 38: 29-59.

17. Vanamail P, Subramanian S, Das PK, Pani SP, Rajagopala PK, Bundy DAP, Grenfell BT, 1989. Estimation of age specific rates of acquisition and loss of Wuchereria bancrofti infection. Trans R Soc Trop Med Hyg 83: 689-693.
18. Srividya A, Pani SP, Rajagopalan PK, Bundy DAP, Grenfell, BT, 1991. The dynamics of infection and disease in Bancroftian filariasis. Trans $R$ Soc Trop Med Hyg 85: 255-259.

19. Chan MS, Guyatt HL, Bundy DAP, Medley GF, 1996. Dynamic models of schistosomiasis morbidity. Am J Trop Med Hyg 55: $52-62$.

20. Medley GF, Bundy DAP, 1996. Dynamic modeling of epidemiologic patterns of schistosomiasis morbidity. Am J Trop Med Hyg 55 (suppl): 149-158.

21. Das PK, Manoharan A, Srividya A, Grenfell BT, Bundy DAP, Vanamail P, 1990. Frequency distribution of Wuchereria bancrofti microfilariae in human populations and its relationships with age and sex. Parasitology 101: 429-434.

22. Day KP, Grenfell B, Spark R, Kazura JW, Alpers MP, 1991 Age specific patterns of change in the dynamics of Wuchereria bancrofti infection in Papua New Guinea. Am J Trop Med Hyg 44: 518-527.

23. Ottesen EA, 1992. Infection and disease in lymphatic filariasis: an immunological perspective. Parasitology 104: S71-S79.

24. Woolhouse MEJ, 1992. A theoretical framework for the immunoepidemiology of helminth infection. Parasite Immunol 14: $563-578$

25. Olszewski W, Jamal S, 1994. Skin bacterial factors in progression of filarial lymphodema. Lymphology 27: 148-149.

26. Pani SP, Yuvaraj J, Vanamail P, Dhanda V, Michael E, Grenfell BT, Bundy DAP, 1995. Episodic adenolymphangitis and lymphodema in patients with bancroftian filariasis. Trans $R$ Soc Trop Med Hyg 89: 72-74.

27. Day KP, Gregory WF, Maizels RM, 1991. Age-specific acquisition of immunity to infective larvae in a bancroftian filariasis endemic area of Papua New Guinea. Parasite Immunol 13: 277-290.

28. Grenfell BT, Michael E, Denham DA, 1991. A model for the dynamics of human lymphatic filariasis. Parasitol Today 7 : 318-323.

29. Michael E, Grenfell BT, Isham VS, Denham DA, Bundy DAP, 1998. Modelling variability in lymphatic filariasis: macrofilarial dynamics in the Brugia pahangi-cat model. Proc R Soc Lond B Biol Sci 265: 155-165.

30. Amaral F, Dreyer G, Figueredo-Silva J, Noroes J, Cavalcanti A, Samico, SC, Santos A, Coutinho A, 1994. Live adult worms detected by ultrasonography in human bancroftian filariasis. Am J Trop Med Hyg 50: 753-757.

31. Freedman DO, 1998. Immunopathogenesis of human lymphatic filariasis. Parasitol Today 14: 229-234.

32. Addiss DG, Dimock KA, Eberhard ML, Lammie PJ, 1995. Clinical, parasitological and immunologic observations of patients with hydrocele and elephantiasis in an area with endemic lymphatic filariasis. J Infect Dis 171: 755-758.

33. Maizels RM, Sartono E, Kurniawan A, Partono F, Selkirk ME, Yazdanbakhsh M, 1995. T-cell activation and the balance of antibody isotopes in human lymphatic filariasis. Parasitol Today 11: 50-57.

34. WHO, 1992. Lymphatic filariasis: the disease and its control. World Health Organ Tech Rep Ser 821.

35. Lindsey JK, 1996. Parametric Statistical Inference. New York: Oxford University Press.

36. Anderson RM, May RM, 1985. Herd immunity to helminth infection and implications for parasite control. Nature 315: 493-496.

37. Rajagopalan PK, Das PK, Subramanian S, Vanamail P, Ramaiah KD, 1989. Bancroftian filariasis in Pondicherry, South India: 1. Pre-control epidemiological observations. Epidemiol Infect 103: 685-692.

38. Pani SP, Balakrishnan N, Srividya A, Bundy DAP, Grenfell BT, 1991. Clinical Epidemiology of Bancroftian filariasis: effect of age and gender. Trans R Soc Trop Med Hyg 85: 260-264.

39. Grenfell BT, Das PK, Rajagopalan PK, Bundy DAP, 1990. Frequency distribution of lymphatic filariasis microfilariae in human populations: population processes and statistical estimation. Parasitology 104: 417-427.

40. Guyatt HL, Bundy DAP, Medley GF, Grenfell BT, 1990. The relationship between the frequency distribution of Ascaris 
lumbricoides and the prevalence and intensity of infection in human communities. Parasitology 101: 139-143.

41. Meyrowitsch DW, Simonsen PE, Makunde WH, 1995. Bancroftian filariasis: analysis of infection and disease in five endemic communities of north-eastern Tanzania. Ann Trop Med Parasitol 89: 653-663.

42. Maizels RM, Lawrence R, 1991. Immunological tolerance: the key feature in human filariasis? Parasitol Today 7: 271-276.

43. Guyatt HL, Bundy DAP, 1991. Estimating prevalence of community morbidity due to intestinal helminths: prevalence of infection as an indication of the prevalence of disease. Trans $R$ Soc Trop Med Hyg 85: 778-782.

44. Chan MS, Medley GF, Jamison D, Bundy D.A.P, 1994. The evaluation of potential global morbidity atttributable to intestinal nematode infections. Parasitology 109: 373-387.

45. Maizels RM, Bundy DAP, Selkirk ME, Smith DF, Anderson RM, 1993. Immunological modulation and evasion by helminth parasites in human populations. Nature 365: 797-805.

46. Lammie PJ, Hitch WL, Allen EMW, Hightower W, Eberhard ML, 1991. Maternal filarial infection as risk factor for infection in children. Lancet 337: 1005-1006.

47. Kar SK, Mania J, 1993. Filarial hydrocele and its treatment with DEC. Prog Lymphology 14: 364-368.

48. Michael E, Bundy DAP, 1998. Herd immunity to filarial infection is a function of vector biting rate. Proc $R$ Soc Lond $B$ Biol Sci 265: 855-860. 